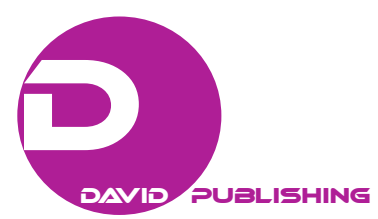

\title{
The Internet and its Psychological and Social Effects on the UAEs Youth
}

\author{
Fawzia Abdulla AlAli \\ Sharjah University, Sharjah, UAE
}

\begin{abstract}
The subject of this research concentrate on indicating psychological and social effects for the Internet on students in Sharjah University/Communication College/in other words the researcher try to know students opinion and impressions regarding the use of Internet and effect of the same, as well as opinion of positive and negative benefits regarding Internet, especially for the university's students who use Internet repeatedly in their daily routine. This stage marked by facts and characteristics based on search for information, explore the students world, and their expectations for that science. This study is the first in its kind in United Arab Emirates that try to shed light on the social and psychological effects of the Internet, therefore we pave the way in front of those who administer Internet and indicate to them guidelines for that to allow decisions maker to be aware about the world around university's student, the world that enjoy less interest for use of Internet at university, and what is the social and psychological effects of the use of Internet. And the study allow providing information and data that several sectors in society need. It draws clear image for the use of Internet at university by the student and whatever social and psychological effects that happened as a result of that use, so it consider as a survey for whom desire to know such information in United Arab Emirates.
\end{abstract}

Keyword: Internet, social effects, psychological effects, adult

\section{Introduction}

Nowadays, the world witnesses a new stage in the field of modern technology developments. Results and conclusions of three revolutions are combined together: The information revolution, means of communication revolution, which is represented in modern communication technologies, and finally, computers revolution, which penetrated all aspects of life and formed part of all means of communication ${ }^{1}$.

Due to availability of Internet, and the continuous desire of using it, Internet users became unable to recognize its concepts or even appreciate its contents. In the eighties, confusion was the most notable obstacles hindering the process of developing Internet, which turned it into an unpractical management. However, through the evolution of new techniques, some developments started to enter into the environment of Internet. This is also the case with services provided by the Internet, through its prominent development recently achieved, which enabled us to communicate and receive different information and services via the Internet

Fawzia Abdulla AlAli, Ph.D., Assistant professor, Teaching Assistant, College of Communication, Sharjah University.

1 Dr. Salwa Al-Awaadi: The ethical dimension in practicing electronic shopping in Egypt, field study on a sample of companies operating in Cairo City. A research submitted for the Conference Internet Revolution and Culture and its Impact-Sharjah "Forum of Youth"-Dr. Nasr Al-Din Al-Ayadi-2004. 
without expensive costs ${ }^{2}$. For instance, the e-mail enabled us to communicate with people from all-over the world cheaply, rapidly, and effectively. Moreover, we are able now to browse all research references (Bibliography) from the Library of Congress, obtain all types of data, make promotions for companies and institutions, access all globally well-known facilities, and these represent a small part of the amazing capabilities of Internet ${ }^{3}$.

The impact of technology, technological information and information in the political, social and economic fields as well as Psychology and security aspects of a society is an extremely great one ${ }^{4}$. Thus, the researcher has chosen to study the customs and methods of using Internet through Sharjah University students-Faculty of Communication - since they are considered part of the most effective categories of population adopting this new phenomenon from both positive and negative perspectives.

\section{Research Problem}

Based on the above-mentioned content, this study attempts to get acquainted with the psychological impacts of Internet through Sharjah University Students/Faculty of Communication/for enabling the researcher to know their viewpoints and impressions regarding this type of use, its impact on them as well as the views of individuals representing both positive and negative sample towards the Internet; especially the university students, who use it repetitively within their daily routine.

In order to achieve these objectives, the researcher attempts to answer the following questions:

- The hours university students spend on Internet.

- Number of students, who have main personal webpages on the Internet.

- The extent to which students are allowed to access Internet in the University.

- Importance of Internet in the life of those students.

- The numerous Internet applications.

- Positive and negative impacts of Internet.

- Social impacts of using Internet.

- Psychological impacts of Internet.

The most important suggestions for supporting positive impacts of using Internet.

\section{Importance of This Research}

This research gains importance from the University Students' importance as being the core of the society and due to the features and facts of this stage, she discusses information in this research and discovers the world of students as well as their expectations regarding this science.

The students' desire to search for different information, to gain various experiences, stems from several sources in which there is an increasing interest in using Internet as a new technique, and hence, the importance of study increases.

This study is considered the first of its type in UAE focusing on the social and psychological impacts of

\footnotetext{
${ }^{2}$ A study on the impact of Internet on the growth of reading, electronic and high skills of female students. Dr. Shaker Abd El-Azim Mohammed Qenawy/Emirates University, 2-Al-Naam Hammadi/Emirates University.

${ }^{3}$ Research thesis presented during the Seminar of Internet Culture and its Impact on Youth in Sharjah 2004 (A research presented to the Ninth Annual Scientific Conference)—Faculty of Mass Communication-Cairo University-Prof. Dr. Ahmed Abd Al-Muqeet, Ethics of Public Relations Practices on Internet, a research submitted to the Ninth Annual Scientific Conference - Cairo University-Faculty of Mass Communication, May 2003 PPs 1046-1047.

${ }^{4}$ Dr. Ashraf Ahmed Abd Al-Muqeet, previous reference, p. 1048.
} 
Internet. Thus, we give the opportunity for those, who are in charge of the Internet with its instructions and the extent of being accustomed to it in order to enable the decision-makers to become well-acquainted with this world, which is linked to the university student since this world gains less attention in terms of using Internet at the university as well as the social and psychological impacts of this use on students. This study allows providing information and data required by several sectors in the society, and it shows the reality of using Internet with all psychological and social impacts caused by it, and as a result, it is considered a collective source for those, who wish to know this type of information in UAE.

\section{Research Approach}

This research is considered one of the descriptive studies, and the research adopts the approach of survey; where a field study is carried out within the framework of survey approach based on choosing a sample consisting of 2002 male and female students from Sharjah University in this study, which was conducted during the year 2013-2014. The sample is divided equally between males and females, but after distributing the questionnaire, the number of sample became 192, with 95 males and 97 females. This category was specially chosen as being the most interested part in using Internet in the field of their specialization since this type of informatory researches in general and public opinion researches is being increasingly used as well as listeners and spectators in particular. In the framework of using the survey approach, it is represented in studying students' opinions and tendencies, which move towards using Internet as well as depending on applying a research form by using the method of personal interview with the research sample for collecting data, analyzing them, and deriving results after that.

\section{Previous Studies}

(1) A study conducted in January 2012 by the University of Minnesota found that $75 \%$ of questioned parents have stated that the Internet improves their method of obtaining parenting related information, $19.7 \%$ found parenting websites too complex to navigate, and $13.1 \%$ of the group did not find any useful parenting information on any website ${ }^{5}$.

Many studies have shown that parents view the Internet as a hub of information especially in their children's education ${ }^{6}$. They feel that there is a positive correlation between the use of the Internet and family conflicts. In conjunction with using the Internet for social means, there is a risk of exposing familial information to strangers, which is perceived to parents as a threat and can ultimately weaken family boundaries.

(2) A report released in October 2012 by Ofcom focused on the amount of online consumption done by children aged 5-15 and 15\% of parents do not take active measures to adequately inform their children of safe Internet browsing; these parents have either spoken only briefly to their children about cautious surfing or do not do anything at all.

When addressing those with lack of parental control over the Internet, parents state that their child is rarely alone (defined for children from 5-11 years old) or that they trust their children when they are online (for children 12-15 years old). Eighty percent of parents ensure that their child has been learned Internet safety from school and $70 \%$ of parents feel that the benefits of using the Internet are greater than the risks that come along

\footnotetext{
5 Omar Sami, Digital Communication Lines for Participants: Dslmailo edition 42162, May 2002, 20 Rabia'a Al-Awal 1423, First Part.

6 Abd Al-Rahman Abd Al-Aziz. "Research Concepts and Techniques-Radio and TV-Researches Magazine-Issued by Al-Azhar University, 1984, p. 11.
} 
with it. ${ }^{7}$

(3) An American study conducted by PewInternet released on November 20, 2012, reveal that parents are highly concerned about the problems the Internet can effect their teenage children. Fourty-seven percent of parents are tend to worry about their children being exposed to inappropriate material on the Internet and $45 \%$ of the parents are concerned about their children's behaviour towards each other both online and offline. ${ }^{8}$

This study is conducted in order to know the social impacts of Internet on the youth in UAE through positive and negative impacts of using Internet. The number of sample is 1000 chosen from all emirates, and the most important results reached in this study are:

- Most male and female youth categories use Internet as well as the age group 19-29 years, which means that the use of Internet is mainly represented in the youth categories since the percentage reached $73.10 \%$ from the total sample of study, and the majority of Internet users are bachelors as their percentage reached $87.10 \%$, followed by the married people category with a percentage of $11.10 \%$.

- It is evident that more than $76.80 \%$ emphasized the easiness of obtaining and exchanging information, which is considered one of the positive impacts of using Internet and communicating with friends inside and outside the country $(62.10 \%)$ as well as increase in the informational, cultural and educational level (58.20\%).

- One of the most negative impacts of using Internet is highly represented in the social impact with a percentage of $64.30 \%$ from the total sample individuals, which is an aspect that should not be ignored in order to put an end to moral corruption in our societies as well as the negative impact on the family life with a notable percentage of $51 \%$. The most important recommendation made by the study is to disseminate awareness and knowledge in terms of using Internet as well as defining and realizing both negative and positive aspects of Internet, and imposing family censorship on sons and daughters, who use Internet.

The study is summarized in knowing the extent of contribution made by Internet regarding the development of some skills; such as reading, and the importance of using them in the life of the majority as well as getting acquainted with the most significant trends of reading in order to consider them when designing future curricula. The study is conducted on a sample of 50 female students, whose ages range from 18-29 years old. The most important results of this study are the following:

- The female students' interests tended towards following the updates of Internet, then scientific commercial subjects and antics come next, then stories and literary subjects, which are given special attention whether via Internet or through library in their traditional form.

- Among all of the results, there is one asserting that the increasing invasion of using the information network (Internet) in the academic and scientific life; where it became a main element by virtue of the facilities provided to the female students as well as the excitement provided through the required subject along with the continuous progress of programs, media, and subjects.

- Guiding and educating youth continuously regarding the dangers of Internet addiction, methods of treatment and Internet usage regulations.

\section{The Reality of Internet Cafés Youth's Usage in UAE_A Field Study}

\footnotetext{
7 "Internet and youth in UAE-Field analytical study: Mohammed Ayesh, Mohammed Qirat, A research presented to The Seminar: Culture and Internet and its Impact on Youth in the Islamic Forum-Impact of Culture and Media-Sharjah University-2004 from pp. 1-50.

8 Youth Internet Addiction-Aspects, Results and Treatment, a research presented for The Islamic Forum in Sharjah on Internet and Youth - prepared by Rami Lotfi Alawy, from pp. 1-14.
} 
The summary of this study is discussing positive and negative impacts of using Internet cafés and revealing the reality of Internet usage in UAE as well as stating its impacts on users in order to get acquainted with social and educational dangers faced by users. The study is conducted on a number of Internet cafés in UAE, and 200 questionnaires were distributed and the study sample was presented. The most important results achieved by the study are as follows:

- $45.00 \%$ of the sample individuals had a range of $1-3$ years of usage, $44.00 \%$ of the sample individuals had a range of 1-2 hours daily and half of the sample used Internet throughout the whole week. The sample individuals describe their monthly expenditure as ranging between 1-49 Dirhams. Results of the study indicated that the great majority used Yahoo, and that the reasons for frequently going to Internet cafés were due to the change of atmosphere, which took the first position, and the most important reasons of using Internet were unseparated.

- The most important recommendations are represented in intensifying censorship on Internet cafés, specifying the age of 18 to be the minimum limit of admitting youth to the Internet cafés.

- Family enlightenment in terms of social and educational dangers of using Internet by youth, the necessity of establishing educational websites targeting the youth category visiting Internet cafés and prohibiting isolation at the Internet cafés by making the use uncovered.

The study is summarized in knowing the religious thoughts presented by some websites; especially everything related to the neutral and scientific Islamic Identity, and knowing whether female students of Education, in particular, interact with these thoughts positively or negatively, the negatives of using Internet with the rest of female students of Education in UAE and the most important recommendations made by the study are as follows:

In light of reality witnessing failure of our educational researches to benefit from technology; especially the Internet, in directing the sound religious culture to our students to face any religious thoughts that contradict Islamic thoughts of our religion and traditions, which are derived from our upright religion, this study gives some suggestions:

(1) A comparative study to know the impact of using Internet - as being one of the technological methods of university education, developing the sound religious concepts for male and female students of Faculty of Education.

(2) A comparative study to know the impact of using Internet - as being one of the technological methods of university education, on the religious awareness of male and female students from different faculties.

(3) Studying the impact of using Internet on students' tendencies towards non-Islamic religious doctrines.

(4) A descriptive, diagnostic, conversational study on obstacles preventing male and female students of Education from using Internet.

(5) A study on the various considerable, challenges, confrontations, reflections, and consequences of using Internet by youth, and knowing the methods and motives of using Internet by youth. The study is conducted on a sample consisting of 516 male and female individuals, and the most important results of this study are as follows: $47.8 \%$ of the sample individuals have been using Internet from two or three years, $77.6 \%$ of the sample individuals use Internet at home, the majority of the sample uses Internet for a period ranging from 1-2 hours daily, and $58.4 \%$ of the sample individuals prefer to use Internet in the evening. The most important purposes, for which Internet is used, are: information (60.3\%), e-mail (58.1\%), assisting in the curriculum $(51.2 \%)$, scientific research (44\%), and phone calls $(44.4 \%) .37 .7 \%$ of the sample individuals do not get 
permission from parents to access the informational network.

The most important recommendations made by this study are: Increasing the level of awareness among youth regarding the nature of the international information network and how to use it positively. Providing alternative uses for the existing inappropriate content, providing specialists in schools and universities to instruct the youth in order to use the information network positively as well as including the subject of Internet within the educational curricula from primary to the university stage.

\section{Conclusion}

Previous studies recommended deepening the theoretical framework in the field of enriching mass media regarding the importance of Internet as a valuable method that can be used in the field of marketing and advertising. This is due to its ability to achieve interaction with audience and to provide a prompt echo return , but none of these studies addressed the importance of Internet for university students, which is the idea discussed by the researcher in her study.

\section{Sample of Study}

A sample of Sharjah University students that reached 200 male and female students is chosen for this study, which was prepared during the academic year 2009. The sample was divided equally between male and female student, and this sample was chosen in particular as being the most interested part in using Internet in its field of specialization.

\section{Specification of Data}

It is decided to select the quality and quantity of data required to be collected from the academic field ${ }^{9}$, which is the focus of this study. After determining the research subject, these data will clarify the different aspects of using Internet, and the required information shall be presented to specialists of information science.

\section{Preparation and Distribution of Questionnaire}

This questionnaire is designed in its initial form based on the previous experience of the researcher in the field of Internet as well as applying the quantitative approach when preparing this research. In addition, this questionnaire has been subject to review by a group of professors and experts in the field of this research due to their insight and acquaintance with the used scientific research approaches in order to achieve the set objectives.

A primary test has been conducted for the questionnaire with a sample consisting of 20 persons with backgrounds similar to those, who were included in the study in order to know the method of posing questions in general and the method of evaluating them in relation to the required approach as well as knowing the questions, which caused difficulty for participants and made them completely unable to answer them. The primary test was followed by a review of the questionnaire in its final form as well as registering and printing the required content into several copies distributed to $10 \%$ of those, who participated in this research. Two weeks after work is finished in this field, it was verified that the work was performed at a high level of quality with a percentage of $85 \%$.

Finally, the questionnaire was given to the 190 students, who participated in this study from the Media

\footnotetext{
${ }^{9}$ Mariam Al-Ali. Previous reference, p. 17, Faculty of Education, The Aspired Reality, 1-10.
} 
Section. There is no doubt that comprehensiveness and exclusivity of this research were maintained during this process, and participants' reactions were collected within two months, March and April 2013.

\section{Research Approach}

This research is considered a descriptive study and the research follows the survey approach ${ }^{10}$; where a field study is conducted within the framework of survey approach, which is based on choosing a sample consisting of 190 students from Faculty of Communication, Sharjah University for this study, which was prepared during the academic year 2013-2014, and the sample was divided equally between both male and female students. This category was chosen, in particular as being the most interested part in using Internet in its field of specialization since this type of media researches, public opinion researches; especially listeners and spectators, is increasingly being used. In the framework of using the survey approach, it is represented in studying students' views and tendencies towards using Internet through depending on applying a research form by using the method of personal interview with the research sample to collect data, which will be analyzed and results shall be concluded later on. ${ }^{11}$

\section{Sample of Study}

A sample of Sharjah University students that reached 190 male and female students is chosen for this study, which was prepared during the academic year 2013-2014. The sample was divided equally between male and female student, and this sample was chosen in particular as being the most interested part in using Internet in its field of specialization.

\section{Specification of Data}

It is decided to select the quality and quantity of data required to be collected from the academic field, which is the focus of this study. After determining the research subject, these data will clarify the different aspects of using Internet, and the required information shall be presented to specialists of information science.

\section{Preparation and Distribution of Questionnaire}

This questionnaire is designed in its initial form based on the previous experience of the researcher in the field of Internet as well as applying the quantitative approach when preparing this research. In addition, this questionnaire has been subject to review by a group of professors and experts in the field of this research due to their insight and acquaintance with the used scientific research approaches in order to achieve the set objectives.

A primary test has been conducted for the questionnaire with a sample consisting of 20 persons with backgrounds similar to those, who were included in the study in order to know the method of posing questions in general and the method of evaluating them in relation to the required approach as well as knowing the questions, which caused difficulty for participants and made them completely unable to answer them. The primary test was followed by a review of the questionnaire in its final form as well as registering and printing the required content into several copies distributed to $10 \%$ of those, who participated in this research. Two

\footnotetext{
${ }^{10}$ A thesis submitted to Youth Internet Addiction-Aspects, Results and Treatment, a research submitted to The Islamic Forum in Sharjah on "Internet and Youth"-prepared by Rami Lotfi Alawy, from pp. 1-14.

11 Mariam Al-Ali. Social Impacts of Using Internet, a research submitted for the Seminar of Internet Culture and its Impact on Youth (2004), p. 14.
} 
weeks after work is finished in this field, it was verified that the work was performed at a high level of quality with a percentage of $85 \%$.

Finally, the questionnaire was given to the 200 students, who participated in this study from the Media Section. There is no doubt that comprehensiveness and exclusivity of this research were maintained during this process, and participants' reactions were collected within two months, November and December 2013.

\section{Field Study}

Table 1

Shows Sample Distribution According to Type

\begin{tabular}{lcc}
\hline Number type & Number & Percentage \\
\hline Males & 95 & $49.4 \%$ \\
Females & 97 & $50.6 \%$ \\
Total & 192 & $100 \%$ \\
\hline
\end{tabular}

The Following is evident from Table 1:

- Results showed that 97 items had a percentage of 50.6\% of the sample individuals from females, whereas the number of males 95 items had a percentage of $49.4 \%$.

- It is apparent through this table that females' interest in using Internet is more than males' interest, which is considered an ordinary result since females in UAE society hang out less often than males do which is a matter that suits customs and traditions since it is non-preferable for females to hang out more than males.

Table 2

Shows the Age Group of the Sample

\begin{tabular}{|c|c|c|c|c|c|c|}
\hline \multirow{2}{*}{ Age group } & \multicolumn{2}{|c|}{ Males } & \multicolumn{2}{|c|}{ Females } & \multicolumn{2}{|c|}{ Total } \\
\hline & Number & $\%$ & Number & $\%$ & Number & $\%$ \\
\hline $17-19$ & 40 & $42.1 \%$ & 50 & $51.4 \%$ & 90 & $46.8 \%$ \\
\hline $19-20$ & 35 & $36.8 \%$ & 35 & $36.0 \%$ & 70 & $36.4 \%$ \\
\hline $21-25$ & 20 & $21.0 \%$ & 120 & $12.4 \%$ & 32 & $16.6 \%$ \\
\hline Total & 95 & $100 \%$ & 97 & $100 \%$ & 192 & $100 \%$ \\
\hline
\end{tabular}

The Following is evident from Table 2:

- Results showed on the males and females level that the number of males from the age group (17-19) with 40 items had a percentage of $42.1 \%$, whereas the number of females with 50 items had a percentage of $51.4 \%$, and the number of males and females was equal at the age group (19-21) since the number of sample individuals for each one 35 items had a percentage of $36.8 \%$ for males against $36.0 \%$ for females.

- Whereas the number of sample individuals at the age group (21-25) with 20 items had a percentage of (21.0\%) against 12 items of females with a percentage of (12.3\%). On the total level, the number of males and females at the age group (17-19) with 90 items had a percentage of $46.8 \%$ and it occupied the first position, and this result was equal to the one of the study conducted by the researcher, Mariam Al-Ali in her research on the social impacts of using Internet with techniques and programs in the university stage. The following age group is (19-21) with a percentage of $36.4 \%$ and it occupied the second position followed by the age group (22-25) with a percentage of $16.6 \%$ and it occupied the third and last position.

- It is evident according to the above-mentioned that males and females at the age group (17-19) are the most interested category to express their opinion about Internet than both the second and last age groups. 
- This indicates that whenever the age group is younger, it is more interested in this type of studies than other categories.

Table 3

Shows the Educational Level of the Sample

\begin{tabular}{|c|c|c|c|c|c|c|}
\hline \multirow{2}{*}{ Specialization } & \multicolumn{2}{|c|}{ Males } & \multicolumn{2}{|c|}{ Females } & \multicolumn{2}{|c|}{ Total } \\
\hline & Number & $\%$ & Number & $\%$ & Number & $\%$ \\
\hline First Year & 45 & $47.3 \%$ & 50 & $51.5 \%$ & 95 & $56.7 \%$ \\
\hline Second Year & 27 & $28.4 \%$ & 25 & $30.9 \%$ & 52 & $27.0 \%$ \\
\hline Third Year & 15 & $15.7 \%$ & 14 & $11.9 \%$ & 29 & $15.1 \%$ \\
\hline Fourth Year & 13 & $13.6 \%$ & 8 & $8.2 \%$ & 23 & $10.9 \%$ \\
\hline Total & 95 & $100 \%$ & 97 & $100 \%$ & 192 & $100 \%$ \\
\hline
\end{tabular}

The Following is evident from Table 3:

- Results showed on the males and females level that $51.5 \%$ of females against $47.3 \%$ of males are in the first year and occupied the first position, followed by a percentage of $30.9 \%$ of females and $28.4 \%$ of males in the second year, and $15.7 \%$ of males against $12.9 \%$ of females in the third year, whereas the percentage in the fourth year reached $13.6 \%$ of males against $8.2 \%$ of females and occupied the last position.

- On the total level, the males and females percentage in the first year reached $56.7 \%$ and occupied the first position followed by the second year with a percentage of $27.0 \%$ and $15.1 \%$ for the third year, and $10.9 \%$ for the fourth year.

Table 4

Shows Sample Distribution According to Scientific Specialization

\begin{tabular}{lcccccc}
\hline \multirow{2}{*}{ Specialization } & \multicolumn{2}{c}{ Males } & \multicolumn{2}{c}{ Females } & \multicolumn{2}{c}{ Total } \\
\cline { 2 - 7 } & Number & $\%$ & Number & $\%$ & Number & $\%$ \\
\hline Audience Communication & 35 & $36.8 \%$ & 50 & $51.5 \%$ & 85 & $44.2 \%$ \\
Arts & 6 & $6.3 \%$ & 25 & $25.7 \%$ & 31 & $16.1 \%$ \\
Sharia'a and Law & 25 & $26.3 \%$ & 10 & $10.3 \%$ & 35 & $18.2 \%$ \\
Science & 5 & $5.2 \%$ & - & - & 5 & $2.6 \%$ \\
Art & - & - & 5 & $5.1 \%$ & 5 & $2.6 \%$ \\
Medicine & 5 & $5.2 \%$ & 3 & $1.6 \%$ & 8 & $4.1 \%$ \\
Engineering & 8 & $8.4 \%$ & 2 & $2.0 \%$ & 10 & $5.2 \%$ \\
Business Administration & 11 & $11.5 \%$ & 2 & $2.0 \%$ & 13 & $6.7 \%$ \\
Total & 95 & $100 \%$ & 97 & $100 \%$ & 192 & $100 \%$ \\
\hline
\end{tabular}

The following is evident from Table 4:

- Results of the study on the males and females level indicated that the percentage of sample participants from the Audience Communication Section was 50 of females against 36.8\% of males and occupied the first position, followed by participants from the Faculty of Arts with a percentage of $25.7 \%$ of females against $6.3 \%$ of males and occupied the second position, participants from the Faculty of Sharia'a and Law occupied the third position, whereas the rest of the specializations reached low percentages that ranged between $2.6 \%$ and $6.7 \%$.

- On the total level, the percentage of Audience Communication was $44.2 \%$ and occupied the first position followed by Sharia'a and Law with a percentage of $18.2 \%$ and occupied the second position followed by Arts 
with a percentage of $16.1 \%$, while percentages of the remaining specializations ranged between $2 \%$ and $6 \%$.

Table 5

Shows Sample Distribution According to its Use of Internet

\begin{tabular}{|c|c|c|c|c|c|c|}
\hline \multirow{2}{*}{ Continuation } & \multicolumn{2}{|c|}{ Males } & \multicolumn{2}{|c|}{ Females } & \multicolumn{2}{|c|}{ Total } \\
\hline & Number & $\%$ & Number & $\%$ & Number & $\%$ \\
\hline Always & 55 & $57.8 \%$ & 60 & $61.8 \%$ & 115 & $59.8 \%$ \\
\hline Sometimes & 35 & $36.8 \%$ & 30 & $30.9 \%$ & 65 & $33.8 \%$ \\
\hline Rarely & 5 & $5.2 \%$ & 7 & $7.3 \%$ & 12 & $6.2 \%$ \\
\hline Total & 95 & $100 \%$ & 97 & $100 \%$ & 192 & $100 \%$ \\
\hline
\end{tabular}

The following is evident from Table 5:

- Results of study, on the males and females level, indicated that the percentage of Internet users from the sample individuals are always females with a percentage of $61.8 \%$ against $57.8 \%$ of males, and the difference between both percentages does not have any statistical significance on the confidence level (95\%), and occupied the first position, whereas the percentage of those who use Internet sometimes was $36.8 \%$ of males against $30.9 \%$ and the difference between both percentages is not statistically significant, and occupied the second position followed by "rarely" with a low percentage for males $(5.21 \%)$ against $7.3 \%$ for females.

- On the total level, it is evident that $59.8 \%$ of sample individuals "always" use Internet and occupied the first position, followed by "sometimes" with a percentage of $33.8 \%$, and the difference between both percentages is not statistically significant, and occupied the second position, whereas "rarely" reached a low percentage of $6.2 \%$.

- This indicates that this new technique is capable of attracting students' attention to it and being interested in using it continuously.

Table 6

Shows the Sample Distribution According to Hours of Using Internet

\begin{tabular}{|c|c|c|c|c|c|c|}
\hline \multirow{2}{*}{ Hours of usage } & \multicolumn{2}{|c|}{ Males } & \multicolumn{2}{|c|}{ Females } & \multicolumn{2}{|c|}{ Total } \\
\hline & Number & $\%$ & Number & $\%$ & Number & $\%$ \\
\hline 1-2 Hours & 14 & $14.7 \%$ & 5 & $5.1 \%$ & 19 & $9.8 \%$ \\
\hline 3-4 Hours & 40 & $42.1 \%$ & 60 & $61.8 \%$ & 100 & $52.0 \%$ \\
\hline 5-6 Hours & 30 & $31.5 \%$ & 20 & $20.6 \%$ & 50 & $26.0 \%$ \\
\hline 7-8 Hours & 5 & $5.2 \%$ & 3 & $3.0 \%$ & 8 & $4.1 \%$ \\
\hline 9 Hours and More. & 6 & $6.2 \%$ & 9 & $9.2 \%$ & 15 & $7.8 \%$ \\
\hline Total & 95 & $100 \%$ & 97 & $100 \%$ & 192 & $100 \%$ \\
\hline
\end{tabular}

The following is evident from Table 6:

- Results of the study, in relation to hours of Internet usage, indicated that on males and females level, the highest percentage of the hours of usage reached $61.8 \%$ for females against $42.1 \%$ for males, and the difference between both percentages does not have any statistical significance, and it occupied the first position followed by the number of hours (5-6) with a percentage of $31.5 \%$ for males against $20.6 \%$ for females, and it occupied the second position, and the difference between both percentages does not have statistical significance at confidence level 95\%, whereas the remaining categories 7-8 and (9 hours and more) had a low percentage that ranged between $6.3 \%$ and $9.2 \%$, which are not high. On the total level, the usage category (3-4 hours) reached $52.0 \%$, and it occupied the first position, followed by the category (5-6 hours) with a percentage of $26.0 \%$, 
which occupied the second position, whereas the remaining categories reached low percentages.

- This indicates that Internet is important to occupy the time of male and female youth since the category of 4-5 hours is not a simple percentage, and the time spent by youth in using Internet indicates their strong enthusiasm and demand for Internet, and this result is similar to the results of other studies conducted in the Arab world in terms of youth's Internet usage, which reached a similar percentage.

Table 7

Shows Sample Distribution According to Places of Internet Usage

\begin{tabular}{|c|c|c|c|c|c|c|}
\hline \multirow{2}{*}{ Places of usage } & \multicolumn{2}{|c|}{ Males } & \multicolumn{2}{|c|}{ Females } & \multicolumn{2}{|c|}{ Total } \\
\hline & Number & $\%$ & Number & $\%$ & Number & $\%$ \\
\hline Home & 45 & $47.3 \%$ & 60 & $61.8 \%$ & 105 & $54.6 \%$ \\
\hline University & 15 & $10.5 \%$ & 37 & $38.1 \%$ & 52 & $27.0 \%$ \\
\hline Café & 35 & $36.8 \%$ & - & - & 35 & $18.2 \%$ \\
\hline Other places & - & - & - & - & - & - \\
\hline Total & 95 & $100 \%$ & 97 & $100 \%$ & 192 & $100 \%$ \\
\hline
\end{tabular}

The following is evident from Table 7:

- Results of the study indicated that places of sample individuals' Internet usage on the males and females level reached the highest percentage of using Internet at home, which was $61.8 \%$ for females against $47.3 \%$ for males, and the difference between both percentages is not statistically significant, and it occupied the first position, followed by using Internet at the university with a percentage of $37.1 \%$ for females against $10.5 \%$ for males, and the difference between both percentages is statistically significant at confidence level $95 \%$, which occupied the second position, while the percentage of using Internet at cafés occupied the third position, with a percentage of $36.8 \%$ for males, whereas there were not any indication stating that females use Internet at cafés.

- On the total level, we find that using Internet at home occupied the first position with a percentage of $54.6 \%$, followed by usage at the university, which occupied the second position with a percentage of $27.0 \%$ and the difference between both percentages is statistically significant at confidence level $95 \%$.

- It is evident that males are the only ones, who use Internet at cafés with a percentage of $18.2 \%$ against nothing for females, and this indicates that the nature of the Emirati society is a conservative one since girls cannot go to Internet cafés as this opposes the dominant customs and traditions of the society.

Table 8

Shows Sample Distribution According to Timings of Internet Usage

\begin{tabular}{llllccc}
\hline \multirow{2}{*}{ Timings of usage } & \multicolumn{3}{c}{ Males } & \multicolumn{2}{c}{ Females } & \multicolumn{2}{c}{ Total } \\
\cline { 2 - 7 } & Number & $\%$ & Number & $\%$ & Number & $\%$ \\
\hline Morning & 25 & $26.3 \%$ & 18 & $18.5 \%$ & 38 & $19.7 \%$ \\
Evening & 50 & $52.6 \%$ & 45 & $46.3 \%$ & 95 & $49.4 \%$ \\
Late at Night & 25 & $26.3 \%$ & 34 & $35.5 \%$ & 59 & $52.0 \%$ \\
Total & 95 & $100 \%$ & 97 & $100 \%$ & 192 & $100 \%$ \\
\hline
\end{tabular}

The following is evident from Table 8:

- Results of study, on males and females level, indicated that in relation to Internet usage timings, percentages were consecutively in the evening $52.6 \%$ for males against $46.3 \%$ for females, and it occupied the first position, and the difference between both percentages is not statistically significant at confidence level 
95\%, followed by the morning, and late at night timings with a percentage of $35.5 \%$ for females against $26.3 \%$ for males, and the difference between both percentages is not statistically significant, and it occupied the second position, whereas morning usage occupied the third position with a percentage of $6.3 \%$ for males against $18.5 \%$ for females, and the difference between both percentages is not statistically significant.

- On the total level, the percentage of late at night usage occupied the first position since it reached 52.0\%, and this result corresponds to the result of the study conducted by Dr. Ahmed Falah Al-Amoushi on the reality of Youth's Usage of Internet Cafés in UAE, which was presented in the Seminar titled The Internet Culture and Its Impact on Youth, followed by night usage with a noticeable difference that reached $49.4 \%$. Whereas, morning usage occupied the last position with a percentage of $19.7 \%$ and the difference between both percentages is statistically significant at confidence level $95 \%$.

- This indicates that family censorship on sons and daughters regarding this usage does not exist since they are left to watch anything late at night, which may be harmful to health and may waste their time as well as leading to mental and physical fatigue.

- This result is similar to the results of studies conducted in different areas of the Arab World.

Table 9

Shows Sample Distribution According to Methods of Usage

\begin{tabular}{|c|c|c|c|c|c|c|}
\hline \multirow{2}{*}{ Methods of usage } & \multicolumn{2}{|c|}{ Males } & \multicolumn{2}{|c|}{ Females } & \multicolumn{2}{|c|}{ Total } \\
\hline & Number & $\%$ & Number & $\%$ & Number & $\%$ \\
\hline Individually & 50 & $52.6 \%$ & 60 & $61.8 \%$ & 110 & $57.2 \%$ \\
\hline Closed & 35 & $36.8 \%$ & 20 & $20.6 \%$ & 55 & $28.6 \%$ \\
\hline Collectively & 10 & $10.5 \%$ & 17 & $17.5 \%$ & 27 & $14.0 \%$ \\
\hline Total & 95 & $100 \%$ & 97 & $100 \%$ & 192 & $100 \%$ \\
\hline
\end{tabular}

The following is evident from Table 9:

- Results of study indicated that the most used Internet usage method was the individual usage with a percentage of $61.8 \%$ for females against $52.6 \%$ for males, and it occupied the first position, and the difference between both percentages does not have statistical significance at confidence level 95\%, whereas the closed usage percentage reached $36.8 \%$ for males and $20.6 \%$ for females, and it occupied the second position, and the difference between both percentages is not statistically significant. In terms of collective usage, it occupied the third and last position for both males and females with a percentage of $17.5 \%$ for females against $10.5 \%$ for males.

- On the total level, it is evident that the most commonly used method is the individual usage method with a percentage of $57.2 \%$, followed by the closed usage with a significant difference that reached $28.6 \%$, whereas the collective usage method reached $14.0 \%$ and it occupied the third and last position.

- Through the above-mentioned data, we conclude that sample individuals use Internet individually, which is due to the freedom of sample individuals in terms of the Internet usage method, and it is also evident that there is extreme freedom represented in the desire of a significant percentage of sample individuals to watch in a closed isolated way. 
Table 10

Shows Sample Distribution According to Exchange Opinions With Friends of Internet

\begin{tabular}{|c|c|c|c|c|c|c|}
\hline \multirow{2}{*}{ Exchange opinions } & \multicolumn{2}{|c|}{ Males } & \multicolumn{2}{|c|}{ Females } & \multicolumn{2}{|c|}{ Total } \\
\hline & Number & $\%$ & Number & $\%$ & Number & $\%$ \\
\hline Yes & 50 & $52.6 \%$ & 25 & $25.7 \%$ & 75 & $39.0 \%$ \\
\hline No & 45 & $47.3 \%$ & 72 & $74.2 \%$ & 117 & $60.9 \%$ \\
\hline Total & 95 & $100 \%$ & 97 & $100 \%$ & 192 & $100 \%$ \\
\hline
\end{tabular}

The following is evident from Table 10:

- The study illustrated that the majority of sample individuals answered "No" in terms of exchanging opinions with friends of Internet, with a percentage of $74.2 \%$ for females against $47.3 \%$ for males, and the difference between both percentages is statistically significant. Concerning those, who answered "Yes", the percentage of males was $52.6 \%$ against $25.7 \%$ for females, and the difference between both percentages is statistically significant at confidence level $95 \%$.

- On the total level, the percentage of those, who exchange opinions with friends about Internet reached $60.9 \%$, and it occupied the first position, followed by the percentage of those, who answered "Yes" (39.0\%). This indicates that the majority of sample individuals do not prefer exchanging opinions with friends regarding what they watch.

Table 11

Shows Sample Distribution According to the Mostly Watched Websites

\begin{tabular}{|c|c|c|c|c|c|c|}
\hline \multirow{2}{*}{ Types of websites } & \multicolumn{2}{|c|}{ Males } & \multicolumn{2}{|c|}{ Females } & \multicolumn{2}{|c|}{ Total } \\
\hline & Number & $\%$ & Number & $\%$ & Number & $\%$ \\
\hline Sports Websites & 35 & $36.8 \%$ & 15 & $15.4 \%$ & 50 & $26.0 \%$ \\
\hline Songs Websites & 20 & $21.0 \%$ & 35 & $36.0 \%$ & 55 & $28.6 \%$ \\
\hline Movies Websites & 10 & $10.5 \%$ & 47 & $48.4 \%$ & 57 & $29.6 \%$ \\
\hline Restricted Websites & 30 & $31.5 \%$ & - & - & 30 & $15.6 \%$ \\
\hline Total & 95 & $100 \%$ & 97 & $100 \%$ & 192 & $100 \%$ \\
\hline
\end{tabular}

The following is evident from Table 11:

- Results of study, in terms of websites watched by the sample on males and females level, indicated that the most important watched websites are the movies websites, with a percentage of $48.4 \%$ for females against $10.5 \%$ for males, and the difference between both percentages had statistical significance at confidence level $95 \%$, and this category occupied the first position, while sports websites reached a percentage of $36.8 \%$ for males against $15.4 \%$ for females, followed by songs websites with a percentage of $21.0 \%$ for males against $36.0 \%$ for females, and the difference between both percentages is not statistically significant. With regard to restricted websites, males were the only ones watching them with a percentage of $31.5 \%$, which is considered a noticeable percentage. It is evident that, since this category watches the restricted websites, there is not any sort of censorship on this category, and it does not have any religious control that prevents it from watching those websites that contradict customs and traditions.

- On the total level, songs websites had a percentage of $28.6 \%$ followed by the movies websites $(29.6 \%)$ with a significant percentage, which occupied the second position, while songs websites reached $28.6 \%$ followed by the restricted websites with a percentage of $15.6 \%$, which occupied the last position. 
Table 12

Shows Sample Distribution According to the Impact of Internet on Family Relations

\begin{tabular}{lcccccc}
\hline \multirow{2}{*}{ Impact of Internet } & \multicolumn{2}{c}{ Males } & \multicolumn{2}{c}{ Females } & \multicolumn{2}{c}{ Total } \\
\cline { 2 - 7 } & Number & $\%$ & Number & $\%$ & Number & $\%$ \\
\hline Leads to Family Isolation & 30 & $31.5 \%$ & 40 & $41.2 \%$ & 70 & $36.4 \%$ \\
Leads to Internet Addiction & 25 & $26.3 \%$ & 35 & $36.0 \%$ & 60 & $31.2 \%$ \\
Leads to continuous search for privacy and & 25 & $26.3 \%$ & 12 & $12.3 \%$ & 37 & $19.2 \%$ \\
confidentiality. & 15 & $15.7 \%$ & 10 & $10.3 \%$ & 25 & $13.0 \%$ \\
Leads to confusion due to family observation & 95 & $100 \%$ & 97 & $100 \%$ & 192 & $100 \%$ \\
Total & &
\end{tabular}

The following is evident from Table 12:

- Results of study on the males and females level, showed the most important impacts of Internet from the perspective of sample individuals since "it leads to isolation", with a percentage of $31.5 \%$ for males against $41.2 \%$ for females, and it occupied the first position, followed by "it leads to Internet addiction" (26.3\%) for males against $36.0 \%$ for females, and it occupied the second position. Concerning the remaining impacts, "leads to continuous search for privacy and confidentiality", it reached $26.3 \%$ for males against $12.3 \%$ for females, and the difference between both percentages is statistically significant.

- Regarding the impact "it leads to confusion due to family observation", the percentage for males reached $15.7 \%$ against $10.3 \%$ for females, and the difference between both percentages is statistically significant.

- On the total level, it is evident that the most important impact of Internet is that "it leads to isolation" since the percentage reached $36.4 \%$, and it occupied the first position, followed by "it leads to Internet addiction" with a significant percentage of $31.2 \%$, and concerning the impact "it leads to continuous search for privacy", the percentage reached $36.4 \%$, and it occupied the first position, followed by "it leads to Internet addiction" with a percentage of $31.2 \%$, and in terms of "it leads to continuous search for privacy", the percentage reached $19.2 \%$ and it occupied the third and last position, and $13.0 \%$ for "it leads to confusion due to family observation".

- We conclude that Internet impact on which sample individuals agreed is "it leads to family isolation", which means that sample individuals may seek Internet through this negative impact, and this is a good initiative since parents become aware of their children through this impact, which will result in some consequences affecting family relations in a way that leads to family breakdown, which is considered something unfamiliar to our Islamic society, which always calls for raising our children in an appropriate Islamic way.

Table 13

Shows Sample Distribution According to the Impact of Contents on Family Relations in Terms of Youth's Perspective Towards the Rest of Family Members

\begin{tabular}{llllllc}
\hline \multirow{2}{*}{ Exchanging opinions } & \multicolumn{2}{c}{ Males } & \multicolumn{2}{c}{ Females } & Total \\
\cline { 2 - 7 } & Number & $\%$ & Number & $\%$ & Number & $\%$ \\
\hline Yes & 55 & $57.8 \%$ & 35 & $30.9 \%$ & 85 & $44.2 \%$ \\
No & 40 & $42.1 \%$ & 67 & $69.0 \%$ & 107 & $55.7 \%$ \\
Total & 95 & $100 \%$ & 97 & $100 \%$ & 192 & $100 \%$ \\
\hline
\end{tabular}

The following is evident from Table 13: 
- Results of study, on males and females level, indicated that the percentage of those, who considered that Internet contents affecting family relations in terms of youth's perspective towards the rest of family members, is $57.8 \%$ for males against $30.9 \%$ for females. Concerning those, who answered "No", the percentage reached $42.1 \%$ for males against $69.0 \%$ for females.

- On the total level, it is evident that the percentage of those, who considered that Internet contents affecting family relations in terms of youth's perspective towards the rest of family members, reached $44.2 \%$ against those, who answered "No" (55.7\%), and the difference between both percentages is not statistically significant at confidence level $95 \%$.

- Hence, we find that there is no difference between those, who answered "Yes" and those, who answered "No" in terms of Internet contents affecting youth's perspective towards the rest of the family.

Table 14

Shows the Extent of Contents Abuse to Roles of Mother, Sister and Woman in General From the Sample's Perspective

\begin{tabular}{llllcrcr}
\hline \multirow{2}{*}{ Exchanging opinions } & \multicolumn{3}{c}{ Males } & \multicolumn{3}{c}{ Females } & \multicolumn{2}{c}{ Total } \\
\cline { 2 - 7 } & Number & $\%$ & Number & $\%$ & Number & $\%$ \\
\hline Yes & 45 & $47.3 \%$ & 20 & $20.6 \%$ & 65 & $33.8 \%$ \\
No & 50 & $52.6 \%$ & 77 & $79.3 \%$ & 127 & $66.1 \%$ \\
Total & 95 & $100 \%$ & 97 & $100 \%$ & 192 & $100 \%$ \\
\hline
\end{tabular}

The following is evident from Table 14:

- Results of study indicated that the percentage of those, who answered "Yes" in terms of their opinion about the extent of Internet contents' abuse against the roles of mother, sister and woman in general, reached $47.3 \%$ for males against $20.6 \%$ for females, and the difference between both percentages is statistically significant, whereas those, who answered "No" had a percentage of $52.6 \%$ against $79.3 \%$.

- On the total level, it is evident that those, who answered "No", had a percentage of $66.1 \%$ for males against $23.8 \%$ for females, and the difference between both percentages is statistically significant.

- This indicates that percentages of males are higher than the ones of females in terms of answering this question, which is considered an ordinary matter since females do not watch what males watch, and therefore, the watched things do not impact females.

Table 15

Shows Reasons of Using Internet

\begin{tabular}{lcccccc}
\hline \multirow{2}{*}{ Reasons of Usage } & \multicolumn{3}{c}{ Males } & \multicolumn{2}{c}{ Females } & \multicolumn{2}{c}{ Total } \\
\cline { 2 - 7 } & Number & $\%$ & Number & $\%$ & Number & $\%$ \\
\hline Listening to songs and music. & 45 & $47.3 \%$ & 45 & $46.3 \%$ & 90 & $46.8 \%$ \\
Entertainment. & 34 & $35.7 \%$ & 35 & $36.0 \%$ & 69 & $35.9 \%$ \\
Searching for Information. & 32 & $34.7 \%$ & 25 & $25.7 \%$ & 57 & $29.6 \%$ \\
Reading newspapers and magazines. & 34 & $35.7 \%$ & 20 & $20.6 \%$ & 54 & $28.1 \%$ \\
Phone calls. & 10 & $10.5 \%$ & 25 & $25.7 \%$ & 35 & $18.2 \%$ \\
Curriculum. & 25 & $26.3 \%$ & 30 & $30.9 \%$ & 55 & $28.6 \%$ \\
Restricted Websites & 35 & $36.8 \%$ & - & - & 35 & $18.2 \%$ \\
Downloading Movies. & 10 & $10.5 \%$ & 25 & $25.7 \%$ & 35 & $18.2 \%$ \\
Downloading Programs. & 15 & $15.7 \%$ & 25 & $25.7 \%$ & 35 & $18.2 \%$ \\
Total & 95 & $100 \%$ & 97 & $100 \%$ & 192 & $100 \%$ \\
\hline
\end{tabular}


The following is evident from Table 15:

- Results of study indicated that the most important reason of using Internet on the males and females level is represented in listening to songs with a percentage of $47.3 \%$ for males against $46.2 \%$ for females, and the difference between both percentages is not statistically significant, and this indicates the agreement of both males and females in terms of listening to songs through Internet usage, and it occupied the first position, followed by the restricted websites in relation to males with a percentage of $36.8 \%$ against zero for females, and it occupied the second position. This means that there is not any family censorship on males in terms of Internet usage since this percentage is considered a significant one to access restricted websites, which contradict ethics and religion as this type of websites lead to violation of customs and traditions as well as tending towards committing sins. Whereas, in the third position, entertainment took place with a percentage of $35.7 \%$ for males against $36.0 \%$ for females, and there is not any essential difference between both percentages.

- This indicates both males and females agreement on using Internet for entertainment, and this may mean the inability of providing other methods of entertainment in the society, which encouraged this category of youth to use Internet for entertainment. In the fourth position, the usage of reading newspapers and magazines took place with a percentage of $35.7 \%$ for males against $20.6 \%$ )for females.

- The remaining reasons of using Internet got low percentages that ranged between $10.0 \%$ and $26.3 \%$.

- On the total level, we find that listening to songs and music occupied the first position since it had a percentage of $46.8 \%$, followed by entertainment with a percentage of $35.9 \%$ and it occupied the second position. Searching for information occupied the third position, and using Internet in the curriculum occupied the fourth position, which indicated the inability of Internet to attract students to use it in the curriculum with a high percentage, and the percentages of the remaining reasons of using internet ranged between $5.2 \%$ and $18.2 \%$, which are considered low ones in comparison with the previous percentages.

Table 16

Shows The Sample's Opinion on Obtaining Parents' Permission to Use Internet

\begin{tabular}{|c|c|c|c|c|c|c|}
\hline \multirow{2}{*}{ Parents' permission } & \multicolumn{2}{|c|}{ Males } & \multicolumn{2}{|c|}{ Females } & \multicolumn{2}{|c|}{ Total } \\
\hline & Number & $\%$ & Number & $\%$ & Number & $\%$ \\
\hline Yes & 37 & $38.9 \%$ & 80 & $82.4 \%$ & 117 & $60.9 \%$ \\
\hline No & 85 & $89.4 \%$ & 17 & $17.5 \%$ & 102 & $53.1 \%$ \\
\hline Total & 95 & $100 \%$ & 97 & $100 \%$ & 192 & $100 \%$ \\
\hline
\end{tabular}

The following is evident from Table 16:

- Results of study on the total level reveal that sample distribution according to its opinion about obtaining parents' permission to use Internet, with "Yes" answers, occupied the first position (60.9\%) against "No" answers $(53.1 \%)$, and the difference between both percentages has essential difference at confidence level 95\%, which indicates contradiction between the content of this table and the content of the previous one in terms of accessing restricted websites since sons and daughters wish to obtain permission from parents to use Internet, but they do not use it the right way that suits customs and traditions.

- On males and females level, the percentage of those, who answered "Yes" in terms of obtaining parents' permission reached $38.9 \%$ for males against $82.4 \%$ for females, indicating females desire to use Internet the right way unlike males. 
Table 17

Shows Sample Distribution According to its Opinion Regarding Parents' Observation to Children

\begin{tabular}{lllllcr}
\hline \multirow{2}{*}{$\begin{array}{l}\text { Parents' observation to } \\
\text { children }\end{array}$} & \multicolumn{2}{c}{ Males } & \multicolumn{2}{c}{ Females } & \multicolumn{2}{c}{ Total } \\
\cline { 2 - 7 } & Number & $\%$ & Number & $\%$ & Number & $\%$ \\
\hline Yes & 35 & $36.8 \%$ & 55 & $56.7 \%$ & 90 & $46.8 \%$ \\
No & 60 & $63.1 \%$ & 42 & $43.2 \%$ & 102 & $53.1 \%$ \\
Total & 95 & $100 \%$ & 97 & $100 \%$ & 192 & $100 \%$ \\
\hline
\end{tabular}

Data of Table 17 indicate:

- On males and females level, that the percentage of those, who answered "Yes" regarding the necessity of parents' observation to their children reached $36.8 \%$ against $56.7 \%$, and the difference between both percentages is statistically significant at confidence level $95 \%$, which indicates the extent of females' commitment to rules and their desire to gain parents' observation when using Internet. Whereas, males expressed weak desire.

- Those, who answered "No", had a percentage of $63.1 \%$ for males against $43.2 \%$ for females, and the difference between both percentages is not statistically significant.

- On the total level, we find that the percentage of those, who answered "No" is $53.1 \%$ against $46.8 \%$ for those who answered "Yes", and the difference between both percentages is not statistically significant at confidence level 95\%, which indicates that males and females of the sample do not have the desire to be committed to parents' observation while using Internet.

Table 18

Shows Sample's Opinion About the Positives of Using Internet

\begin{tabular}{lcccccc}
\hline \multirow{2}{*}{ Positives of usage } & \multicolumn{3}{c}{ Males } & \multicolumn{2}{c}{ Females } & \multicolumn{2}{c}{ Total } \\
\cline { 2 - 7 } & Number & $\%$ & Number & $\%$ & Number & $\%$ \\
\hline Escaping from problems & 65 & $68.4 \%$ & 47 & $48.4 \%$ & 112 & $58.3 \%$ \\
Entertainment & 35 & $36.8 \%$ & 65 & $67.0 \%$ & 100 & $52.0 \%$ \\
Communicating with Friends. & 55 & $57.8 \%$ & 25 & $25.7 \%$ & 80 & $41.6 \%$ \\
Obtaining Information. & 30 & $31.5 \%$ & 35 & $36.0 \%$ & 65 & $33.8 \%$ \\
Alternative of Mass Media. & 25 & $26.3 \%$ & 20 & $21.0 \%$ & 45 & $23.4 \%$ \\
Downloading Programs without cost. & 30 & $31.5 \%$ & 35 & $36.8 \%$ & 65 & $33.8 \%$ \\
Total & 95 & $100 \%$ & 97 & $100 \%$ & 192 & $100 \%$ \\
\hline
\end{tabular}

The following is evident from Table 18:

- Results of study on the total level indicated that the percentage of males' opinion about the positives of using Internet represented in "escaping from problems", occupied the first position with a percentage of 58.3\%. Entertainment occupied the second position with a percentage of $52.0 \%$, and communicating with friends occupied the third position with a percentage of $41.6 \%$, followed by obtaining information and downloading programs without cost with equal percentages of $23.8 \%$. Whereas, "alternative of traditional mass media" occupied the last position with a percentage of $23.4 \%$, which indicated the sample's inability to dispense with the traditional methods of using Internet for obtaining news and information.

\section{Results and Suggestions of Study}

There are the results and suggestions of the study:

(1) Results of study showed that the most age group using Internet is the category 19-21 with a percentage of $(46.8 \%)$. 
(2) And that the most important academic year of using Internet is the first year with a percentage of $56.7 \%$, which is considered a normal result since it applies to the age of Internet users that we mentioned previously.

(3) It is evident that a percentage of 59.8\% uses Internet permanently, and it occupied the first position, followed by "sometimes" with a percentage of $33.8 \%$, and this indicates the ability of Internet to attract the sample individuals. ${ }^{12}$

(4) Hours of Internet usage ranged between (3-4) hours with a percentage of $52.0 \%$, which is considered a long period of time since it affects other activities required to be performed by the student as well as affecting academic achievement.

(5) The study revealed that the most important place in which the sample prefers to use Internet is "home", with a percentage of $54.6 \%$, which is considered an ordinary matter since home is the most common place for a human being to stay at; especially for females.

(6) The most common periods of using Internet is the "late at night period" with a percentage of $52.0 \%$ on males and females level, which is considered a significant percentage since the student's Internet usage during this period affects his/her health, academic achievement and daily activity.

(7) It is evident that the highest percentage if Internet usage is "individually" with a percentage of $57.2 \%$ and this reveals the sample's desire to seek privacy when using Internet.

(8) It is evident through results of the study that the percentage of those, who answered "Yes" to "exchange opinions with friends about Internet" reached 39.0\% against those, who answered "No" (60.9\%), which indicates that the sample prefers privacy even in terms of exchanging opinions on using Internet.

(9) The study illustrated that the most important websites accessed by the males of sample individuals are "movies websites" with a percentage of $29.6 \%$, followed by "songs websites" with a percentage of $28.6 \%$ and "sports websites" with a percentage of $29.6 \%$. Whereas, restricted websites were only accessed by males and not by females, with a percentage of $15.6 \%$, which indicates females' commitment to customs and traditions as well as the religion that prevents them from committing sins.

(10) The study illustrated that the most important impacts of Internet from the sample's perspective are represented in the fact that "it leads to family isolation" with a percentage of $36.4 \%$, followed by "Internet addiction" with a percentage of $31.2 \%$. Whereas, "seeking privacy" reached 19.2\%, and "confusion" reached 13.2\%.

(11) It is evident that those, who found that contents used in Internet affect family relations, had a percentage of $44.2 \%$ while those, who found that they do not affect reached $55.7 \%$.

(12) A percentage of $66.1 \%$ of the sample individuals found that those contents used in Internet in terms of the roles of mother, sister and women in general do not affect, which indicates the extent of sample individuals devotion to ethics and religion since what they did not watch did not affect their perspectives regarding mother, sister and women in general.

(13) Results of study indicated that the most important reasons of using Internet by the sample individuals are "listening to songs", which reached $46.8 \%$, followed by "entertainment" with a percentage of $35.9 \%$.

(14) Results of study revealed that $60.9 \%$ of sample individuals answered "Yes" in terms of their opinion to obtain parents' permission to use Internet, which indicates good ethics of the sample individuals.

(15) A percentage of $46.8 \%$ of sample individuals found that there is a necessity for parents to observe their children when using Internet and this result caused the following one represented in the fact that the sample

12 “Children and Parents: Media Use and Attitudes Report”. Ofcom. 23 October 2012. Retrieved 10 February 2013. 
answered "Yes" in terms of obtaining parents' permission since it prefer parents' observation to their children when using Internet.

(16) Some of the most important positives of using Internet are represented in "escaping from problems", which reached $58.3 \%$, followed by "entertainment" with a percentage of $52.0 \%$ and "communicating with friends" with a percentage of $41.6 \%$.

\section{Recommendations}

- Family enlightenment in terms of the social dangers and consequences of using Internet; especially knowing that it is evident through results of the study that the most important reason of using it focuses on "escaping from problems", which reached 58.3\%, which means that the sample individuals suffer from problems, which the family knows nothing about.

- Family's participation with children in showing the importance and benefits of using Internet as well as explaining to them that Internet can be used in beneficial matters other than listening to songs by females. It reached a percentage of $46.8 \%$ and occupied the first position.

- The researcher recommends continuous censorship on children's Internet usage since it is proven that a percentage of $88.2 \%$ for males uses Internet to access restricted websites, which led them to commit sins.

- Parents must attempt to participate with children in watching websites sometimes since the sample expressed its desire to have observation when using Internet.

- Parents must specify fixed hours for using Internet and not to leave Internet provided to them all the time since most of the sample individuals illustrated that they use Internet (3-4) hours daily, which is considered a high average that refers to the importance of time spent by students on Internet.

- Sample individuals found that the most important impact of Internet was represented in the fact that "it leads to family isolation", which indicates the importance of avoiding continuous routine of parents in order not to cause family isolation.

- Participation of the different authorities through setting social awareness programs via the Internet to serve families and the society as well as preparing a report stating the negative effects of Internet on children and the society as a whole.

- Conducting more researches and studies on youth and Internet in order to address all educational, entertaining and social aspects of Internet as well as revealing their advantages and disadvantages for the audience in order to be well-acquainted with them.

\section{References}

Abd Al-Rahman, A. A. (1984). Radio and TV: Research approaches and techniques. Researches Magazine (p. 64), Al-Azhar University.

Abdo, N. (1997). Informatics tomorrow. Computer Science, Communication and Electronics, 25, 41, 75, 77, 101.

Abu Arqub, E. (1993). Human communication and its role in social interaction. Oman: Al-Majd International Publishing and Distributing House.

Al-Abd, A., \& Al-Ghali, F. (1994). Traditions and methods of students' acquaintance with satellite channels. Researches Magazine (p. 344), Al-Azhar University.

Ghoneim, J. (1997). Towards Arabic Internet. Middle East House, 312, 56.

Hassan, S. M. (1983). Media researches: Fundamentals and principles (p. 39). Cairo: Famous Books.

Hatem, A. (1998). Internet in the Middle East. Al-Khaleej Magazine (p. 8).

Madden, M., Cortesi, S., Gasser, U., Lenhart, A., \& Duggan, M. (2012). Parents, teens, and online privacy. Pew Research Center. 